\title{
A versatile cold-molecule collider
}

\author{
Superconducting magnets have been used to trap cold oxygen molecules and study their collisions. This method could lead \\ to a better understanding of low-temperature interactions for a broad range of molecules. SEE ARTICLE P.189
}

\section{DAJUN WANG}

$\mathrm{O}$ n page 189 , Segev et al. ${ }^{1}$ report that they can observe collisions between trapped oxygen molecules at low (sub-kelvin) temperatures, without using the conventional technique of laser cooling. They achieve this feat by directly slowing a fast-moving molecular beam and subsequently loading the molecules into a trap generated by electric currents flowing in superconducting magnets. For this method to work, the only requirement is that the molecules have a magnetic dipole moment, which means that they behave like tiny bar magnets. This condition is much less stringent than those for other techniques ${ }^{2}$ because there are plenty of such molecules in nature. Therefore, the authors' achievement makes it possible to trap many types of molecule for detailed inspection, to understand the interactions and reactions of these molecules at low temperatures.

Much of the contemporary understanding of chemical reactions was obtained from studying molecular collisions ${ }^{3}$ using a device known as a crossed-molecular-beam apparatus ${ }^{4}$. This device causes two well-controlled beams of atoms or molecules to intersect, and can then be used to detect and track the reaction products. However, it is difficult to use such a device to investigate molecular collisions at low temperatures. Cold, and thus slow-moving, molecules take a long time to come into contact with one another. But the beams move at supersonic velocities, which means that molecules from each beam remain in the same region only briefly. Cold-molecule collisions are best studied in a trap, in which the molecules can stay together and interact with each other over a much longer period.

A key feature of a molecular beam is that the molecules move at nearly the same, albeit extremely high, velocity. Because the temperature of a molecule is just the manifestation of how fast it moves, the high velocity of the beam means that the temperature associated with the uniform movement of the molecules is also high. However, this uniform movement implies that the temperature corresponding to the relative motion of the molecules is extremely low. It follows that samples of cold molecules can be obtained by slowing down the beam.

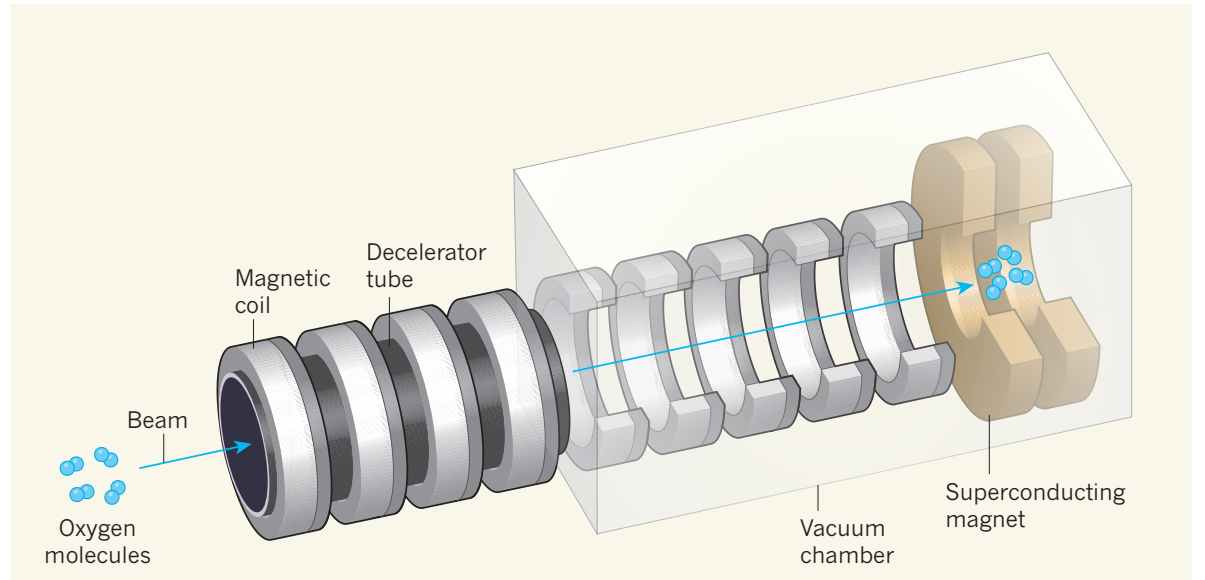

Figure 1 | A method for cooling and trapping molecules. Segev et al. ${ }^{1}$ report experiments in which oxygen molecules in the form of a high-velocity beam travel through a structure known as a decelerator tube and then through a vacuum chamber. As the molecules propagate, they are greatly decelerated by pairs of magnetic coils (light and dark grey). The resulting slow-moving, and therefore cold, molecules enter a magnetic trap that is generated by two superconducting magnets. The molecules remain at the centre of this trap for up to a minute, which enables their collisions to be observed. (Adapted from Fig. 1 of the paper ${ }^{1}$.)

Segev and colleagues did just that. Building on what their research group had previously demonstrated ${ }^{5}$, the authors directed a highvelocity beam of oxygen molecules through a series of pairs of magnetic coils (Fig. 1). By repeatedly switching the electric current in these coils on and off at precise times, the authors generated a varying magnetic field that moved with the beam. During each cycle of on-and-off switching, the molecules received a small momentum 'kick' and slowed down a little. After many of these cycles, the beam velocity was substantially reduced from hundreds of metres per second to only several metres per second - low enough for the molecules to be loaded into a stationary trap.

However, before trapping could occur, the oxygen molecules needed a strong final kick to make them stop at the centre of the trap; otherwise, they would have quickly passed through it. This is where the superconducting magnets came in. Segev and colleagues constructed these magnets using superconducting tapes that were cooled to $15 \mathrm{~K}$ - about one-sixth of the temperature below which the tape materials become superconducting. Such a low operational temperature was key to ensuring that the materials remained in the superconducting phase during the rapid switching of large currents that was needed to stop the slowed molecular beam at the centre of the trap.

Although the entire deceleration process reduced the overall velocity of the beam, it could not decrease the relative velocity between the molecules. The authors therefore needed to run high currents through the superconducting magnets to generate a strong magnetic trap. Because superconducting materials have zero electrical resistance, these currents flowed through the magnets without producing heat, which would cause the surfaces of the magnets to emit gaseous molecules. As a result, the trap could be operated for a long time (almost one minute) without compromising the required vacuum conditions.

By maintaining a good vacuum, Segev et al. observed the signature of two-body molecular collisions: a clear, non-exponential decay in the number of trapped molecules. Without such a vacuum, this signature would be masked by collisions between oxygen molecules and background gas molecules. 
The observation of two-body collisions is a prerequisite for investigating many types of molecular dynamics. One of the most intriguing prospects is the possibility of initiating evaporative cooling ${ }^{6}$, whereby even lower temperatures than that achieved by the authors could be obtained by removing high-energy molecules from the trap. Unfortunately, this process remains to be demonstrated. Moreover, the present work suggests that efficient evaporative cooling might not be feasible in the case of oxygen molecules, because many of the collisions reduce the number of trapped molecules without lowering the temperature.

Segev and colleagues managed to trap a large number of molecules (about one billion) at a high density. This condition, together with the long lifetime of the trap, is indispensable for pushing cold-molecule research forwards. Until now, such conditions have never been achieved simultaneously without the use of laser cooling. The authors' work should therefore be viewed as yet another landmark in this field.

In the past few years, other methods for producing cold molecules have also made breakthroughs ${ }^{7}$. For example, an exotic state of matter known as a quantum degenerate gas, comprising ground-state molecules, has been made by assembling cold atoms ${ }^{8}$. And single molecules have been loaded into traps called optical tweezers with the help of direct laser cooling of ground-state molecules ${ }^{9}$. None of these methods, including the one reported by Segev and colleagues, can be applied to all types of molecule. But putting them all together, a wide range of cold molecules will become available for study. After more than two decades of hard effort, it is now time to start exploring the great promise of cold molecules in many fundamental and practical applications ${ }^{2,7}$.

Dajun Wang is in the Department of Physics, The Chinese University of Hong Kong, Shatin, Hong Kong, China.

e-mail:djwang@cuhk.edu.hk

1. Segev, Y. et al. Nature 572, 189-193 (2019).

2. Carr, L. D., DeMille, D., Krems, R. V. \& Ye, J. New J. Phys. 11, 055049 (2009).

3. Levine, R. D. Molecular Reaction Dynamics Vol. 10 (Cambridge Univ. Press, 2009).

4. Herschbach, D. R. in Advances in Chemical Physics (ed. Ross, J.) Ch. 9 (Wiley, 1966).

5. Akerman, N. et al. New J. Phys. 17, 065015 (2015).

6. Masuhara, N. et al. Phys. Rev. Lett. 61, 935-938 (1988).

7. Bohn. J. L., Rey. A. M. \& Ye, J. Science 357, 1002-1010 (2017).

8. De Marco, L. et al. Science 363, 853-856 (2019).

9. Anderegg, L. et al. Preprint at https://arxiv.org/ abs/1902.00497 (2019).

\section{Flower power as human cancer cells compete}

Cells compete for survival during development. It emerges that mammalian cells on a path to form a tumour express specific versions of the protein Flower when they vie for survival with surrounding normal cells. SEE LETTER P.260

\section{YASUYUKI FUJITA} n multicellular organisms, cells usually communicate with each other in a peaceful manner. But harmony is shattered if survival with normal cells in a process called cell competition. This process was identified in the fruit fly Drosophila melanogaster ${ }^{1}$, and it also occurs in mammals ${ }^{2}$. When cancer starts to form in mammals, competition occurs between normal cells and those on a path to tumour formation, but how the molecular differences between such cells trigger cell competition is poorly understood. Madan et al. ${ }^{3}$ report on page 260 that whether competing mammalian cells win such a battle depends on which version of a membrane protein called Flower they express.

Earlier work ${ }^{4}$ in D. melanogaster revealed that, during cell competition, the cells that survive (winner cells) are distinguished from the cells that die (loser cells) by the version of Flower protein (FWE) that they express. Different versions of this protein are made when the messenger RNA that encodes FWE undergoes a process called alternative splicing. Loser cells express a version of FWE termed FWE ${ }^{\text {Lose }}$ and winner cells express a version termed $\mathrm{FWE}^{\mathrm{Win}}$. When loser cells come into contact with winner cells, the former cells die and the
$\mathrm{I}$ abnormal cells appear and battle for space and latter divide to fill the empty space left by this cell death ${ }^{4}$. An encounter between cells that express FWE ${ }^{\text {Lose }}$ and those that express $\mathrm{FWE}^{\mathrm{Win}}$ is required for cell death to occur because cells that express FWE $\mathrm{L}^{\text {Lose }}$ survive if cells expressing $\mathrm{FWE}^{\mathrm{Win}}$ are absent ${ }^{4}$. However, whether a comparable FWE system functions in mammals was unknown.

Madan and colleagues report that alternative splicing generates four versions of human FWE, which they term FWE1, FWE2, FWE3 and FWE4. Using human breast cancer cells grown in vitro, the authors examined whether any of these versions of FWE behave as winner or loser forms of the protein. They report that, when human cells that express either FWE1 or FWE3 are cultured with cells that express either FWE2 or FWE4, cells that express FWE1 or FWE3 die and those that express FWE2 or FWE4 divide to compensate for the loss of the dying cells. Thus, in this system, cells that express FWE1

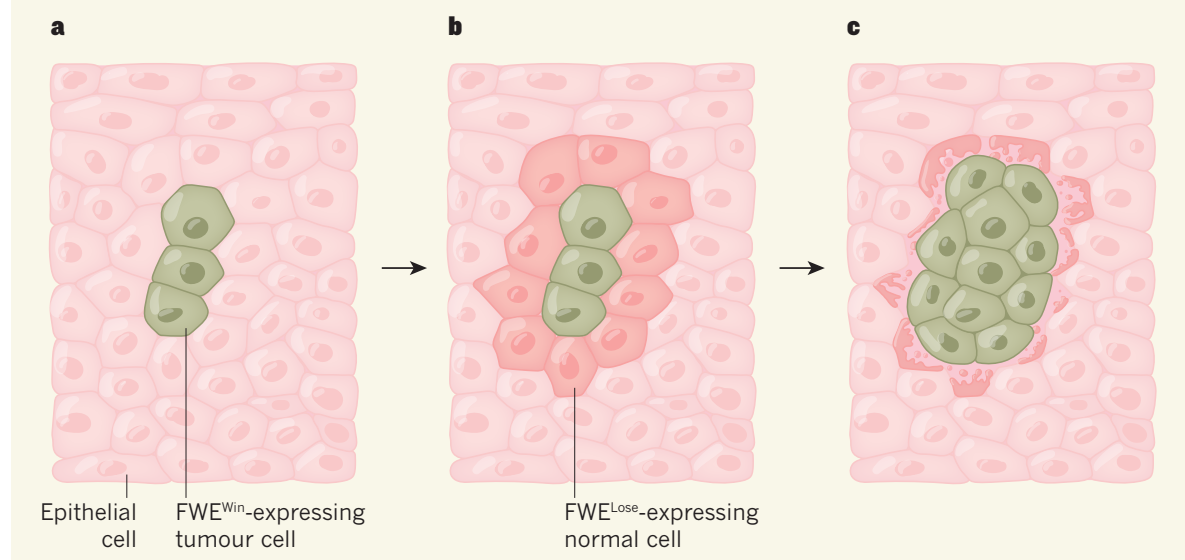

Figure 1 | Mammalian cell competition is driven by the expression of Flower protein. Different versions of a membrane protein called Flower (FWE) are made through a process called alternative splicing. These different versions are termed winner $\left(\mathrm{FWE}^{\text {Win }}\right)$ or loser $\left(\mathrm{FWE}^{\mathrm{Lose}}\right)$, and they affect whether a cell survives or dies depending on which version of FWE is expressed by neighbouring cells. Madan et al. ${ }^{3}$ studied human FWE in a type of cell called an epithelial cell, and investigated normal cells and cancer cells. Their results are consistent with the following model. a, Tumour cells express $\mathrm{FWE}^{\mathrm{Win}}$. b. This leads, through an unknown mechanism, to the expression of $\mathrm{FWE}^{\mathrm{Lose}}$ in neighbouring normal cells. c, Competition between cells expressing $\mathrm{FWE}^{\mathrm{Win}}$ or $\mathrm{FWE}^{\text {Lose }}$ results in the death of $\mathrm{FWE}^{\text {Lose }}$-expressing cells, and the $\mathrm{FWE}^{\mathrm{Win}}$-expressing cells divide to fill the gap in the tissue that arises from this cell death. 